\title{
Análisis de las habilidades psicológicas en los deportistas promesas y talentos guipuzcoanos Analysis of the psychological skills of promising athletes and talents from Gipuzkoa
}

\author{
Amaia Ramírez Muñoz*, Joel Manuel Prieto Andreu* \\ *Universidad Internacional de la Rioja
}

\begin{abstract}
Resumen. Afrontar con éxito el proceso de transición en la consolidación del talento deportivo es fundamental para adaptarse y lograr una estabilidad en el deporte de alto nivel. Las habilidades psicológicas adquieren una gran importancia en este periodo, ya que mediatizan el rendimiento deportivo. El objetivo de esta investigación ha sido analizar las habilidades psicológicas de los deportistas promesas y talentos guipuzcoanos. La muestra estuvo compuesta por la totalidad de los 146 deportistas integrantes del programa de Desarrollo del Talento Deportivo (DTD) de la Diputación Foral de Gipuzkoa. El instrumento utilizado para evaluar las habilidades psicológicas fue el cuestionario de Características Psicológicas Relacionadas con el Rendimiento Deportivo (CPRD). Los resultados obtenidos indican la existencia de diferencias estadísticamente significativas en función del género $(\mathrm{p}<, 05)$ en todas las variables psicológicas analizadas: control de estrés (C.E), influencia de la evaluación de rendimiento (I.E.R), motivación (M), habilidad mental (H.M) y cohesión de equipo (C.EQ). No se evidenció ninguna relación significativa entre las habilidades psicológicas y la edad, pero sí en función de la categoría del deportista (promesa / talento): los deportistas promesas mostraron una menor H.M ( $<<, 05)$ que los talentos deportivos.

Palabras clave: habilidades psicológicas, desarrollo del talento deportivo, transición deportiva, género y edad.
\end{abstract}

\begin{abstract}
Successfully facing the transition process in the consolidation of sports talent is fundamental to adapt to the highest level and achieve stability in the sports career. Psychological skills become very important in this period, as they mediate sports performance. The aim of this study has been to analyse the psychological skills of promising athletes and talents from Gipuzkoa. The sample consisted of all 146 athletes who were members of the Provincial Council of Gipuzkoa for the development of sports talent. The instrument used to evaluate the psychological skills was the questionnaire of Psychological Characteristics Related to Sports Performance. The results obtained indicate that there are statistically significant differences between men and women $(p<, 05)$ in all the psychological variables analyzed: stress control, influence of performance assessment, motivation, mental ability and team cohesion. Likewise, differences were found in mental ability $(\mathrm{p}<, 05)$ between promising athletes and talents, while no statistically significant relation was found between the age and the psychological skills of the sample.
\end{abstract}

Key words: psychological skills, sports talent, sports transition, gender and age.

\section{Introducción}

El talento deportivo ha generado un gran interés en los últimos años y se ha convertido en el foco de atención de muchas investigaciones (Serra-Olivares, Prieto-Ayuso, Pastor-Vicedo y González-Víllora, 2019; Johnston, Wattie, Schorer y Baker, 2018; Coutinho, Mesquita y Fonseca 2016; Torreblanca-Martínez Arráez, Otero-Saborido y GonzálezJurado, 2017; Farinola, Tuñon, Laiño, Marchesich y Pérez, 2018). Pero los resultados obtenidos no son concluyentes, y su estudio se ha visto envuelto en una gran controversia que dificulta el acuerdo entre los investigadores (Lorenzo, Lorenzo y Jiménez, 2015). El avance en el conocimiento de la conceptualización del talento deportivo requiere de investigaciones y estudios realizados desde un enfoque multidisciplinar e integrador (Phillips, Davids, Renshaw y Portus, 2010), dinámico e interaccionista (Lorenzo, et al., 2015). Dinámico, porque las características y habilidades del deportista van evolucionando y consolidándose a lo largo del tiempo, e interaccionista, porque dicha evolución está sometida a la interacción entre las características del deportista y su contexto.

Recogiendo lo anterior, los modelos de transición deportiva (Stambulova, 2003; Wylleman, Reints y Knop, 2013) resultan una aproximación apropiada para el estudio del desarrollo del talento deportivo. Estos modelos equiparan de manera metafórica la carrera deportiva con una carrera vital (Torregrossa, Chamorro y Ramis, 2016), y asumen que el

Fecha recepción: 01-04-20. Fecha de aceptación: 03-08-20

Amaia Ramírez Muñoz

amaia.ramirez@unir.net deportista atraviesa por distintas etapas de transición en su desarrollo que debe ir superando para llegar a la élite deportiva y mantenerse de manera estable. La etapa de transición de consolidación del talento deportivo, es decir, cuando el deportista pasa de ser deportista promesa a talentoso, es un periodo crítico y de gran exigencia psicológica (Morris, Tod y Eubank, 2017; Morris, Tod y Oliver, 2016). A menudo, esta fase suele coincidir con el paso de la categoría junior a senior, y muchos de los deportistas reconocen que es la etapa más difícil y que han fracasado al afrontarla (Stambulova, Alfermann, Statler y Côté, 2009). Para adaptarse a esta nueva etapa, el deportista deberá perfeccionar, incrementar o modificar sus estrategias cognitivas y comportamentales. Un buen desarrollo de las habilidades psicológicas en ese periodo, permitirá al deportista afrontar con mayores garantías de éxito la presión de la competición (Pazmiño, Ucha, García y Pazmiño, 2018).

El desarrollo del talento es por tanto, un proceso complejo, multifactorial y dinámico. Su exhibición, mantenimiento y sostenibilidad va a depender de la interacción de tres factores principalmente: el factor genético, el entorno deportivo y las habilidades psicológicas.

La importancia de las habilidades psicológicas en el rendimiento deportivo ha sido demostrada por un gran número de investigadores (Abdullah, Musa, Maliki, Musawi, Kosni y Suppiah, 2016; Castilla y Ramos, 2012; López-Gullón et al., 2011; MacNamara, Button y Collins, 2010; Weinberg y Gould, 2014). Y han sido consideradas como un factor mediador entre las capacidades físicas, técnicas y tácticas de los deportistas (Mahamud, Tuero y Márquez, 2007) y por lo tanto, en el Desarrollo del Talento Deportivo (DTD). Pero las habi- 
lidades psicológicas de un deportista pueden fluctuar a lo largo del tiempo en función del contexto y la situación (Musculus y Lobinger 2018), por lo que deben de ser evaluadas sistemáticamente para su control. Centrándonos en el periodo de transición objeto de estudio en esta investigación, su evaluación adquiere una gran relevancia, ya que permite establecer hipótesis acerca de la intervención psicológica más apropiada para favorecer el rendimiento deportivo (Abenza, González, Reyes, Reyes y Blas, 2014; Gimeno, Buceta y Pérez-Llantada, 2007; Olmedilla, Ortega, Andreu y Ortín, 2010; Olmedilla, Ortega, Boladeras, Ortín, y Bazaco, 2013), facilitar la manifestación y consolidación del talento, y evitar el abandono deportivo temprano (Gimeno, Buceta y Pérez-llantada, 2001) en las personas con altas capacidades deportivas.

Finalmente, aunque el talento no tiene género, existe cierta controversia con respecto a la existencia de diferencias en las habilidades psicológicas entre los y las deportistas. Según algunos autores, los hombres puntúan más alto que las mujeres en las variables C.EQ (Arias, Cardoso, Aguirre y Arenas, 2016; Hidalgo, Martín y Chirosa, 2015) y en la I.E.R (Medrano y Mateos, 2017). Sin embargo, otros investigadores afirman que tanto hombres como mujeres obtienen puntuaciones similares en la medida de habilidades psicológicas para el rendimiento deportivo (Kruger y Pienaar, 2014; Robbani y Dimyati, 2019).

El presente estudio recoge como objetivo general estudiar las variables psicológicas implicadas en la etapa de transición de consolidación del talento deportivo. Analizar en profundidad las características psicológicas en este periodo y desarrollar baremos propios de esta población específica permitirá desarrollar itinerarios del DTD de mayor calidad que atiendan las diferencias individuales y tengan en cuenta sus especificidades para facilitar que el talento se desarrolle y se consolide con las mayores garantías de éxito. Por lo tanto, se planteó una primera hipótesis afirmando que existen diferencias de género en las habilidades psicológicas de los deportistas evaluados. Una segunda hipótesis sosteniendo que, los deportistas de mayor edad obtendrían mejores puntuaciones en habilidades psicológicas que los deportistas más jóvenes. Y una tercera y última hipótesis, que mantiene que los deportistas talentos, obtendrán una mayor puntuación en las habilidades psicológicas que los deportistas promesas.

\section{Método}

\section{Diseño}

El diseño utilizado en la investigación fue de corte transversal-retrospectivo, descriptivo y correlacional (Thomas y Nelson, 2007). Las variables objeto de estudio fueron: habilidades psicológicas del deportista, género, edad y categoría (promesa o talento deportivo).

\section{Participantes}

Para la realización de esta investigación se ha tomado como muestra la totalidad de los deportistas promesas y talentos del programa de DTD de la Diputación foral de Gipuzkoa correspondientes a la temporada 2017-2018. Un $48,6 \%$ fueron mujeres y un $51,4 \%$ hombres con edades com- prendidas entre 12 y 20 años (con una media de 17 años y una desviación estándar de 1,64), que representaban a 22 modalidades deportivas diferentes (Tabla 1). En total, la muestra estuvo compuesto por 146 deportistas, de los cuales, 124 fueron designados deportistas promesas, y 22 deportistas talentos. Los criterios de inclusión de la muestra fueron haber sido propuesto como deportista promesa y cumplir los criterios especificados en la convocatoria para tal fin $(\mathrm{O}$. 0202/095/2017, de 19 de mayo).

\begin{tabular}{|c|c|c|c|c|c|}
\hline \multirow{2}{*}{+2} & \multicolumn{2}{|c|}{ Talento } & \multicolumn{2}{|c|}{ Promesa } & \multirow[t]{2}{*}{ Tota } \\
\hline & Hombre & Mujer & Hombre & Mujer & \\
\hline Equitación & 0 & 0 & 0 & 1 & 1 \\
\hline Trial & 0 & 0 & 1 & 1 & 2 \\
\hline Halterofilia & 1 & 0 & 0 & 1 & 2 \\
\hline Atletismo & 1 & 2 & 9 & 17 & 29 \\
\hline Hockey hielo & 1 & 0 & 5 & 2 & 8 \\
\hline Tiro con arco & 0 & 0 & 0 & 2 & 2 \\
\hline Remo & 0 & 0 & 8 & 5 & 13 \\
\hline Bádminton & 0 & 1 & 0 & 3 & 4 \\
\hline Baloncesto & 0 & 1 & 2 & 1 & 4 \\
\hline Ciclismo & 0 & 0 & 9 & 6 & 15 \\
\hline Escalada & 0 & 0 & 1 & 0 & 1 \\
\hline Balonmano & 3 & 2 & 2 & 2 & 9 \\
\hline Esquí & 1 & 0 & 2 & 0 & 3 \\
\hline Gimnasia rítmica & 0 & 0 & 0 & 2 & 2 \\
\hline Golf & 0 & 0 & 2 & 3 & 5 \\
\hline Natación & 0 & 0 & 4 & 6 & 10 \\
\hline Judo & 0 & 0 & 3 & 1 & 4 \\
\hline Pelota & 0 & 0 & 2 & 1 & 3 \\
\hline Piragüismo & 1 & 2 & 6 & 6 & 15 \\
\hline Tenis de mesa & 1 & 0 & 1 & 1 & 3 \\
\hline Waterpolo & 0 & 0 & 3 & 4 & 7 \\
\hline Surf & 1 & 1 & 1 & 1 & 4 \\
\hline Total & 10 & 9 & 61 & 66 & 146 \\
\hline
\end{tabular}

\section{Procedimiento}

Esta investigación se realizó dentro del programa de DTD de la Diputación Foral de Gipuzkoa (2017-2018). Las bases del programa fueron publicadas en el BOG del día 26 de mayo de 2017. Para poder ser designado deportista promesa, los participantes tuvieron que rellenar una instancia en la que certificaban el cumplimiento de los requisitos especificados y aceptar voluntariamente participar en este programa (O. 0202/095/2017, de 19 de mayo). Una vez obtenido el compromiso voluntario, y tras comprobar la veracidad de los datos aportados, se aprobó la relación de deportistas admitidos en el programa y se convocó un comité de expertos que evaluó las candidaturas y seleccionó a los 22 deportistas talentosos que mostraron mayores puntuaciones en sus capacidades para el alto rendimiento en base a los siguientes criterios: pruebas físicas, informe técnico de las federaciones, y nivel de compromiso del deportista. De este modo resultaron un total de 124 deportistas promesas y 22 talentos deportivos. El comité de expertos estuvo formado por la directora de deportes de la diputación foral de Gipuzkoa, un experto en ciencias de la actividad física y el deporte, una experta en psicología deportiva, un representante de las federaciones deportivas de Gipuzkoa, y el director de servicio de la dirección de deportes de la diputación. Concluido el nombramiento, se procedió a la realización del cuestionario CPRD vía on-line. El tiempo aproximado para realizar el cuestionario fue de 15 minutos, y para ello se habilitaron unas claves y una página web a la que el deportista debía acceder para su cumplimentación.

\section{Instrumentos}

El CPRD desarrollado por Gimeno et al., (2001) que evalúa las aptitudes psicológicas para el rendimiento deportivo, es el instrumento más utilizado en España cuando se trata de 
valorar el aspecto psicológico de los/as deportistas. El cuestionario tiene un total de 55 ítems con un formato de respuesta tipo Likert con cinco puntos y está compuesto por cinco escalas: a) Control de Estrés (C.E), abarca las características de la respuesta del deportista en relación con las demandas del entrenamiento y la competición (e.g. concentración) y las situaciones potencialmente estresantes que pueden provocar estrés y donde es necesario la presencia de control; b) Influencia de la Evaluación del Rendimiento (I.E.R), abarca las características de la respuesta del deportista ante situaciones en las que éste u otras personas evalúan su rendimiento (e.g. perder la concentración); c) Motivación (M), hace referencia a la motivación por superarse día a día, el establecimiento de metas y la importancia del deporte en relación con otras actividades; d) Habilidad Mental (H.M), comprende habilidades psicológicas que favorecen el rendimiento deportivo (e.g. establecimiento de objetivos); y e) Cohesión de Equipo (C.EQ), define la integración del deportista en su equipo o grupo deportivo. El CPRD ha reportado propiedades psicométricas de fiabilidad y validez en la reunión de varios estudios, como lo demuestra la compilación de Gimeno y Buceta (2010) y otras revisiones específicas (Gimeno, et al., 2010, 2001; López-López, Jaenes-Sánchez, y Cárdenas-Vélez, 2013). El cuestionario ha mostrado utilidad aplicado a deportistas de 13 años de edad en adelante, y su consistencia interna arroja un coeficiente alfa de Cronbach de .85 para todo el instrumento, lo cual lo señala como valor satisfactorio y confiable para su aplicación (Gimeno y PérezLlantada, 2010). En el presente estudio se obtuvo un coeficiente de Cronbach de .91 .

\section{Análisis estadístico}

Para conocer las características psicológicas de la población de deportistas promesas y talentos guipuzcoanos, se calcularon las puntuaciones percentiles de los participantes en cada una de las escalas del CPRD y se realizó un estudio descriptivo de las diferentes variables propuestas en esta investigación. Se aplicó la prueba de normalidad KolmogorovSmirnov para comprobar la normalidad de las variables del cuestionario. Se utilizó el estadístico U de Mann-Withney para analizar las diferencias entre los grupos en función del género y la categoría (promesa/talento deportivo). La relación entre las puntuaciones obtenidas en el CPRD y la edad se analizó utilizando el coeficiente bivariado de Spearman. Se estableció un nivel de significación de $\mathrm{p}<.05$ y el análisis estadístico se realizó usando el Paquete Estadístico para Ciencias Sociales SPSS para Windows v.24.0

\section{Resultados}

En primer lugar, se ha realizado un análisis de los estadísticos descriptivos teniendo en cuenta las puntuaciones percentiles como se puede observar en la Tabla 2.

Así mismo, se han calculado los valores promedio, desviaciones típicas y valores mínimos y máximos obtenidos para cada dimensión del cuestionario CPRD en función del género (Tabla 3).

Se destaca que en general, los deportistas promesas y talentos guipuzcoanos muestran unas altas puntuaciones en todas las habilidades psicológicas evaluadas y los hom-
Tabla 2.

Baremo de puntuaciones centiles en deportistas promesas y talentos guipuzcoanos hombres $y$ mujeres

\begin{tabular}{|c|c|c|c|c|c|c|c|c|c|c|c|}
\hline & \multicolumn{5}{|c|}{ Hombres } & \multicolumn{5}{|c|}{ Mujeres } & \\
\hline$P$ & C.E & I.E.R & M & H.M & C.EQ & C.E & I.E.R & M & H.M & C.EQ & $P$ \\
\hline 10 & 69,20 & 38,20 & 28,00 & 31,00 & 17,40 & 51,80 & 34,00 & 24,60 & 28,00 & 12,60 & 10 \\
\hline 20 & 74,40 & 44,00 & 29,40 & 32,00 & 24,00 & 58,20 & 37,00 & 28,20 & 30,00 & 20,00 & 20 \\
\hline 30 & 77,00 & 47,00 & 31,00 & 33,00 & 25,00 & 63,00 & 39,00 & 30,00 & 31,00 & 21,80 & 30 \\
\hline 40 & 79,80 & 47,80 & 32,00 & 35,00 & 26,00 & 68,40 & 43,40 & 31,00 & 32,40 & 23,40 & 40 \\
\hline 50 & 83,00 & 49,00 & 33,00 & 35,00 & 27,00 & 72,00 & 45,00 & 32,00 & 34,00 & 25,00 & 50 \\
\hline 60 & 86,00 & 50,00 & 34,00 & 36,20 & 27,00 & 76,00 & 47,00 & 32,00 & 34,00 & 26,00 & 60 \\
\hline 70 & 88,40 & 52,40 & 35,00 & 37,00 & 28,00 & 78,00 & 48,00 & 33,00 & 36,00 & 27,00 & 70 \\
\hline 80 & 91,00 & 54,00 & 36,00 & 38,00 & 29,00 & 81,00 & 51,00 & 35,00 & 37,00 & 28,00 & 80 \\
\hline 90 & 93,80 & 56,00 & 37,80 & 39,00 & 29,00 & 90,40 & 54,00 & 37,00 & 38,00 & 29,00 & 90 \\
\hline
\end{tabular}

Tabla 3.

Medias de los constructos del cuestionario CPRD y su relación con la variable género

\begin{tabular}{llccccc}
\hline \multirow{2}{*}{ C.E } & & $\mathrm{N}$ & Media & SD & Mínimo & Máximo \\
& Hombre & 71 & 81,38 & 10,95 & 38 & 98 \\
& Mujer & 75 & 71,23 & 13,28 & 46 & 100 \\
& Total & 146 & 76,16 & 13,18 & 38 & 100 \\
\hline I.E.R & Hombre & 71 & 48,48 & 6,41 & 32 & 60 \\
& Mujer & 75 & 44,29 & 7,14 & 30 & 60 \\
& Total & 146 & 46,33 & 7,09 & 30 & 60 \\
\hline M & Hombre & 71 & 32,82 & 3,67 & 24 & 40 \\
& Mujer & 75 & 31,27 & 4,48 & 15 & 40 \\
& Total & 146 & 32,02 & 4,17 & 15 & 40 \\
\hline \multirow{2}{*}{ H.M } & Hombre & 71 & 35,11 & 3,39 & 25 & 41 \\
& Mujer & 75 & 33,12 & 4,11 & 18 & 40 \\
& Total & 146 & 34,09 & 3,89 & 18 & 41 \\
\hline C.EQ & Hombre & 71 & 25,46 & 4,45 & 10 & 30 \\
& Mujer & 75 & 22,77 & 6,59 & 0 & 30 \\
& Total & 146 & 24,08 & 5,79 & 0 & 30 \\
\hline Nota: C.E & $=$ Cohesión de equipo; I.E.R = Influencia de la evaluación del rendimiento; $\mathrm{M}=$
\end{tabular}

Nota: $\mathrm{C} . \mathrm{E}=$ Cohesión de equipo; $\mathrm{I}$.E.R $=$ Influencia de la evaluación
Motivación; $\mathrm{H} . \mathrm{M}=$ Habilidad mental; $\mathrm{C} . \mathrm{EQ}=$ Cohesión de equipo

bres obtienen puntuaciones más altas en todas las escalas del CPRD. Para valorar si las diferencias halladas en el estudio descriptivo resultan estadísticamente significativas se utilizó la prueba U de Mann-Witnney. Los resultados obtenidos confirman la presencia de diferencias estadísticamente significativas entre hombres y mujeres en los cinco factores evaluados por el CPRD obteniendo las mujeres una menor puntuación en C.E $(Z=-4,81, p<0.01)$, I.E.R del rendimiento, $(Z=-3,60, p<0.01), M(Z=-2,01, p<0.05), H . M(Z=-2,93, p$ $<0.01)$ y C.EQ $(Z=-2,88, p<0.01)$. Es decir, en todas las escalas del instrumento utilizado.

Por otra parte, se realizó un análisis de correlación de Spearman para analizar si existía una relación entre la edad de los deportistas y las puntuaciones obtenidas en las diferentes escalas del CPRD. No se encontraron relaciones estadísticamente significativas en ninguna de las habilidades psicológicas evaluadas.

Finalmente, al estudiar las diferencias en las aptitudes psicológicas para el alto rendimiento en función de la categoría (promesa o talento), la única diferencia estadísticamente significativa encontrada fue la variable H.M $(Z=-2,32, p<$ $0.05)$. De manera que los deportistas promesas obtuvieron una puntuación más baja en esta variable que los deportistas seleccionados como talentos. Para descartar que la edad pudiera estar influyendo en la diferencia de resultados encontrados en la H.M entre promesas y talentos, se utilizó el estadístico U de Mann-Witnney y se comparó la media de edad entre ambos grupos, pero no se encontraron diferencias estadísticamente significativas entre ellos.

\section{Discusión}

De acuerdo al objetivo general planteado, en este estudio se analizaron las habilidades psicológicas, y se obtuvieron las puntuaciones medias y centiles de la totalidad de los deportistas promesas y talentos guipuzcoanos. Los resultados obtenidos en esta investigación arrojan mayores puntuaciones en todas las habilidades psicológicas evaluadas 
que las presentadas por los autores Gimeno y Buceta (2010) en sus estudios de referencia. Tales discrepancias pueden ser debidas a que las características específicas de la muestra de este estudio responden a un contexto, unas características personales y una situación muy específica. Previo a ser aceptados en el programa, todos los deportistas evaluados han demostrado y certificado una gran experiencia deportiva: selecciones nacionales, competiciones internacionales, etc., un alto potencial deportivo y un alto nivel de rendimiento en todo su itinerario. Se trata pues de un contexto muy específico y orientado a la alta competición, y de deportistas de un potencial y un nivel de rendimiento diferente al de la muestra del estudio de referencia, hecho que puede justificar las diferencias encontradas. Estos resultados, están en la línea de los aportados por Musculus y Lobinger (2018), quienes confirmaron que las habilidades psicológicas fluctúan a lo largo del desarrollo deportivo en función del contexto, la persona y la situación, y sugieren la necesidad de evaluar de manera sistemática estas tres variables, para garantizar el desarrollo de las habilidades psicológicas del deportista de manera estable.

Atendiendo a la primera hipótesis planteada, se pudo conocer que existen diferencias de género en las habilidades psicológicas, y que los hombres obtuvieron mayores puntuaciones en todas las escalas evaluadas (C.E; I.E.R; M; H.M; y C.EQ). Estos resultados coinciden parcialmente con los hallados en otros estudios (Medrano y Mateos, 2017; Padilla y Arenas, 2016) en los que los hombres obtuvieron mayores puntuaciones en la variable I.E.R (Medrano y Mateos, 2017) y en la escala M (Padilla y Arenas, 2016) que las mujeres. Sin embargo, contrastan con los hallazgos de otros investigadores. En los estudios realizados por Kruger y Pienaar (2014) y Robbani y Dimyati (2019), los y las deportistas obtuvieron puntuaciones similares en todas las escalas de las habilidades psicológicas evaluadas. Las posibles discrepancias entre los resultados reportados en la literatura y los observados en el presente trabajo pueden ser explicados por diferentes factores como el nivel de competición, la edad y los instrumentos utilizados. Como resalta Prieto (2017) quizá en algún tipo de deporte, las variables deportivas como el nivel de competición o la experiencia deportiva, podrían tener influencia en el rendimiento deportivo, pudiendo ofrecer información adicional que pudiera resultar importante en cuanto a la variación en variables psicológicas como la ansiedad y la motivación. En esta investigación, la totalidad de la muestra eran deportistas de alto rendimiento que competían en el máximo nivel, mientras que en el estudio de Padilla y Arenas (2016), los y las deportistas eran juveniles y seniors, y competían a nivel regional. Por otra parte, en cuanto al tipo de instrumento utilizado, Kruger, y Pienaar (2014), utilizaron el Athletic Coping Skills Scale-28 (Smith, Schutz, Smoll, y Ptacek, 1995), y Robbani y Dimyati (2019) evaluó las habilidades psicológicas mediante el The Psychological Skills Inventory for Sports (PSIS-R-5) de Mahoney, Gabriel y Perkins, (1987). Las diferencias encontradas en los diferentes estudios pudieran verse afectadas por el de instrumento utilizado, y sugieren la necesidad de controlar tanto el tipo de instrumento, como el nivel de competición y la edad a la hora de inferir los resultados.

En cuanto a la relación entre edad y habilidades psicoló- gicas, y haciendo referencia a la segunda de las hipótesis planteadas, en este estudio no se ha encontrado relación estadísticamente significativa entre la edad y ninguna de las escalas del CPRD. Los resultados obtenidos en la revisión de la bibliografía consultada y los estudios previos realizados revelan cierta controversia con respecto a dicha hipótesis. Por ejemplo, Moreno, Cervelló y González-Cutre, (2006) encontraron que los deportistas de menor edad presentan mayores niveles de motivación. Sin embargo, Coimbra et al, (2013), midieron la motivación a través de la motivación extrínseca y de ego, indicando que los deportistas de mayor edad presentan una mayor motivación que los más jóvenes. Los resultados obtenidos en esta investigación confirman los obtenidos por otros autores que utilizaron el CPRD para evaluar las habilidades psicológicas (Medrano y Mateos, 2017), y afirman no encontrar diferencias en la motivación de los deportistas en función de la edad. Las razones de esta falta de concordancia en los resultados pudiera deberse a las diferencias metodológicas de los estudios realizados. Por otra parte, la falta de relación entre la edad y las habilidades psicológicas pudiera ser explicada por las características específicas de esta muestra. Los deportistas promesas y talentos, son aquellos que se han ajustado exitosamente a cada una de las transiciones en su desarrollo deportivo, y comparten todos una misma etapa de transición. El desarrollo de las habilidades psicológicas pudiera no estar relacionado con la edad cronológica, sino con la la experiencia deportiva lo que pudiera estar mediatizando las habilidades psicológicas adquiridas por el deportista.

Finalmente, la tercera de las hipótesis planteada en esta investigación afirmaba que los deportistas talentosos obtendrían una mayor puntuación en las habilidades psicológicas que los deportistas promesas. Esta hipótesis solo se pudo corroborar parcialmente ya que sólo se encontraron diferencias estadísticamente significativas en el factor de H.M del CPRD. No es casual que sea esta variable la que diferencia a los deportistas promesas de los más talentosos, ya que incluye competencias psicológicas como son el establecimiento de objetivos, el análisis objetivo del rendimiento, el ensayo conductual en imaginación y la autorregulación (Gimeno, et al., 2001) que favorecen el rendimiento deportivo. Los resultados obtenidos confirman que los deportistas de mayor potencial que reflejan un nivel superior, poseen también unas mejores habilidades psicológicas para el alto rendimiento (Pazmiño, et al., 2018; Gimeno, et al., 2007). Otros investigadores también han encontrado diferencias en las habilidades la importancia del factor psicológico en el rendimiento deportivo (Abdullah, et al., 2016; Castilla y Ramos, 2012; López-Gullón et al., 2011; MacNamara, et al., 2010; Weinberg y Gould, 2014). Poseer una buena habilidad mental puede ejercer una gran influencia sobre los resultados del deportista, y muy especialmente en la proyección de posibles futuros talentos, ya que incide directamente sobre la planificación y la optimización de los recursos propios para afrontar con éxito cada una de las diferentes etapas de transición en el itinerario de desarrollo del deportista. Pero lo que en este trabajo se subraya, es que precisamente en esta etapa de transición de deportista promesa a talentoso que muchos deportistas describen como la más difícil y reconocen no haber afrontado con éxito (Stambulova, et al. 2009), la 
H.M adquiere una gran importancia en el desarrollo y consolidación del talento deportivo.

Entre las limitaciones de este trabajo, podemos mencionar que no se han controlado alguna de las variables personales o del entorno del deportista que puedan estar mediatizando las puntuaciones obtenidas en las habilidades psicológicas como son el momento de la competitivo, el número de horas de dedicación al entrenamiento y la competición o el haber padecido alguna lesión recientemente. De cara a futuras investigaciones, sería interesante tener en cuenta dichas variables, además de realizar investigaciones con una mayor amplitud geográfica que permitan la obtención de baremos nacionales en esta franja de población. Contar con baremos específicos de referencia en esta etapa de transición, así como la realización de estudios longitudinales que permitan analizar las fluctuaciones de las habilidades psicológicas, facilitaría el control de la planificación del itinerario del DTD en jóvenes con altas capacidades y resultaría de gran interés para los entrenadores y psicólogos que los acompañan.

\section{Conclusiones}

El periodo de transición durante el que se consolida el talento deportivo, supone una de las etapas más difíciles en la trayectoria de un deportista.

Las habilidades psicológicas entre hombres y mujeres difieren de manera estadísticamente significativa, por lo que se deben de tener en cuenta por parte de los técnicos y los psicólogos deportivos a la hora de planificar los planes de intervención y de acompañamiento en el desarrollo del talento deportivo.

No existe relación entre la edad y las habilidades psicológicas, por lo que resulta necesaria la evaluación sistemática de estas habilidades para su control.

La habilidad mental, es decir, la capacidad de establecer objetivos, realizar un análisis objetivo del rendimiento, el ensayo conductual en imaginación, así como la autorregulación cognitiva, son las habilidades psicológicas que mayor relación tienen con un ajuste adecuado en esta etapa de transición de consolidación del talento deportivo.

Evaluar y adquirir unas buenas habilidades psicológicas permite adaptarse y afrontar con éxito las exigencias y demandas competitivas durante esta fase de transición.

\section{Agradecimientos}

Este trabajo ha sido realizado gracias al apoyo, la buena disposición y el impulso ofrecido por la Dirección de Deportes de la Diputación Foral de Gipuzkoa y en especial por su directora Goizane Álvarez Irijoa.

\section{Referencias}

Abdullah, M., Musa, R., Maliki, A. B., Musawi, H., Kosni, N., y K. Suppiah, P. (2016). Original Article Role of psychological factors on the performance of elite soccer players, 16, 170 176. doi:10.7752/jpes.2016.01027

Abenza, L., González, J., Reyes, L., Reyes, F., \& Blas, A. (2014).

Descripción y evaluación del entrenamiento psicológico de una deportista de regata clase laser radial. Revista Iberoamericana de Psicología del Ejercicio y del Deporte/ : 9, 1, 2014. Las Palmas de Gran Canaria: Universidad de Las Palmas de Gran Canaria, Servicio de Publicaciones. doi:10.1400/218736

Arias, I., Cardoso, T., Aguirre, H. y Arenas, J. (2016). Características psicológicas de rendimiento deportivo en deportes de conjunto. Psicogente, 19(35), 25-36. doi:10.17081/ psico.19.35.1206

Coimbra, D. R., Gomes, S. S., Oliveira, H. Z., Rezende, R. A., Castro, D., Miranda, R., \& Filho, M. G B. (2013). Características motivacionais de atletas brasileiros [Motivational characteristics of Brazilian athletes]. Motricidade, 9(4), 64 72. doi:10.6063/motricidade.9(4).1179

Coutinho, P., Mesquita, I., \& Fonseca, A. M. (2016). Talent development in sport: A critical review of pathways to expert performance. International Journal of Sports Science \& Coaching, 11(2), 279-293. doi:10.1177/1747954116637499

Farinola, M. G., Tuñón, I., Laiño, F., Marchesich, M., y Pérez Rodríguez, M. (2018). Perfil socioeducativo y económico de deportistas adolescentes de élite argentinos. Retos: Nuevas Tendencias En Educación Física, Deporte y Recreación, 2041(34), 172-176. Recuperado de https://cutt.ly/ FdEr4TU

Fenoy Castilla, J., \& Campoy Ramos, L. (2012). Sport performance, leadership styles and experimental avoidance in young footballers from Almeria. Revista De Psicologia Del Deporte, 21(1), 137-142. Recuperado de https://cutt.ly/BdEtftE

Fradejas Medrano, E., \& Espada Mateos, M. (2017). Evaluación de la motivación en adolescentes que practican deporte en edad escolar (Evaluation of motivation in teenagers who practice sports in school age). Retos, (33), 27-33. Recuperado de https://cutt.ly/edEtFc3

García Pazmiño, M., García Ucha, F., Arévalo García, N., \& García Pazmmiño, S. (2018). Presiones Deportivas y Disposición Óptima Combativa (Sports Pressures and Optimal Combative Disposition). Retos, (35), 335-340. Recuperado de https:// cutt.ly/tdEt1QY

Gimeno-Marco, F. \& Pérez-Llantada-Rueda, M. C. (2010). Características psicométricas del cuestionario CPRD. En GimenoMarco, F. \& Buceta, J. M. (Edits.). Evaluación psicológica en el deporte: cuestionario de «características psicológicas relacionadas con el rendimiento deportivo» (CPRD) (p. 81 124). Madrid: Dykinson.

Gimeno, F., Buceta, J. M., \& Pérez-Llantada, M. C. (2007). [The influence of psychological variables on sports performance: assessment with the Questionnaire of Sports Performancerelated Psychological Characteristics]. Psicothema, 19(4), 667-672. Recuperado de https://cutt.ly/HdEyttR

Gimeno, F., Buceta, J. M., y Pérez-Llantada, M. D. C. (2001). El cuestionario «características psicológicas relacionadas con el rendimiento deportivo» (CPRD): Características psicométricas. Análise Psicológica, 19(1), 93-113. doi.10.14417/ap.346

Hidalgo, L. Martín, I. y Chirosa, L.J. (2015). Análisis de las características psicológicas y la toma de decisiones en un grupo de nadadores. Revista iberoamericana de psicología del ejercicio y el deporte, 10, 49-56.

Johnston, K., Wattie, N., Schorer, J., \& Baker, J. (2018). Talent Identification in Sport: A Systematic Review. Sports medicine (Auckland, N.Z.), 48(1), 97-109. doi:10.1007/s40279-0170803-2

Kruger,A., \& Pienaar,A. E. (2014). Gender differences in the sport psychological skills profile of adolescent sport participants/ 
: original research. International SportMed Journal, 15(4), 474-482. Recuperado de https://journals.co.za/content/ismj/ 15/4/EJC164092

López-Gullón, J., García-Pallares, J., Berengüi Gil, R., MartínezMoreno, A., Morales-Baños, V., Torres-Bonete, M., \& Díaz, A. (2011). Physical and psychological factors in predicting olympic wrestling performance. Revista De Psicologia Del Deporte, 20(2), 573-588. Recuperado de https://www.rpdonline.com/article/view/817/789

López-López, I. S., Jaenes-Sánchez, J. C., \& Cárdenas-Vélez, D. C. (2013). Adaptación para futbolistas (CPRD-F) del cuestionario «características psicológicas relacionadas con el rendimiento deportivo» (CPRD) [Adaptation for footballer (CPRD-F) of «Psychological Characteristics Related to the Sport Performance» questionnaire (CPRD)]. Cuadernos de Psicología del Deporte, 13(2), 21-30. doi:10.4321/S157884232013000200003

Lorenzo, A. (2001). Hacia un nuevo enfoque del concepto de talento deportivo. Revista de Entrenamiento Deportivo, 15(2), 27-33.

Lorenzo, A., Lorenzo, J., y Jiménez, S. (2015). Y si nos olvidamos de la detección del talento... Y si individualizamos el proceso de desarrollo de su talento $=$ And if we forget the talent detection... And if we individualize the development of HIS Talent,21, 105-127. doi:10.14201/aula201521105127

MacNamara, A., Button, A. y Collins, D. (2010). The role of psychological characteristics in facilitating the pathway to elite performance part 1: Identifying mental skills and behaviors. The Sport Psychologist, 24(1), 52-73. doi:10.1123/ tsp.24.1.52

Mahamud, J., Tuero, C., \& Márquez, S. (2007). Características psicológicas relacionadas con el rendimiento: comparación ente los requerimientos de los entrenadores y la percepción de los deportistas. Revista De Psicologia Del Deporte, 14(2). Recuperado de https://www.rpd-online.com/article/view/183

Mahoney, M. J., Gabriel, T. J., \& Perkins, T. (1987). Psychological Skills and Exceptional Athletic Performance, The Sport Psychologist, 1(3), 181-199. Recuperado de https:// journals.humankinetics.com/view/journals/tsp/1/3/articlep181.xml

Moreno, J. A., Cervelló, E., \& González-Cutre, D. (2006). Motivaciónautodeterminada y flujo disposicional en el deporte. Anales de Psicología, 22(2), 310-317.

Morris, R., Tod, D., y Eubank, M. (2017). From youth team to first team: An investigation into the transition experiences of young professional athletes in soccer. International Journal of Sport and Exercise Psychology, 15(5), 523-539. doi:10.1080/ 1612197X.2016.1152992

Morris, R., Tod, D., y Oliver, E. (2016). An Investigation Into Stakeholders' Perceptions of the Youth-to-Senior Transition in Professional Soccer in the United Kingdom. Journal of Applied Sport Psychology, 28(4), 375-391. doi.10.1080/ 10413200.2016.1162222

Musculus, L., y Lobinger, B. H. (2018). Psychological characteristics in talented soccer players - Recommendations on how to improve coaches' assessment. Frontiers in Psychology, 9(FEB), 1-6. doi:10.3389/fpsyg.2018.00041

Olmedilla Zafra, A., Ortega Toro, E., Boladeras Esteve, A., Ortiìn MonteroF. J., y Bazaco Belmonte, M. J. (2013). Entrenamiento en estrategias y teìcnicas psicoloìgicas y percepcioin de ayuda en futbolistas juveniles. SPORT TK-Revista EuroAmericana De Ciencias Del Deporte, 2(1), 51-58. doi:10.6018/185751
Olmedilla Zafra, A., Ortega, E., Andreu, M., \& Ortín, F. (2010). Entrenamiento de habilidades psicológicas en futbolistas: evaluación de eficacia. Revista De Psicologia Del Deporte, 19(2), 249-262. Recuperado de https://www.rpd-online.com/ article/view/471

Padilla, I. A., y Arenas, J. A. (2016). Características psicológicas de rendimiento deportivo en deportes de conjunto * Psychological characteristics sports performance in team sports, 19(c), 25-36. doi:10.17081/psico.19.35.1206

Phillips, E., Davids, K., Renshaw, I., \& Portus, M. (2010). Expert Performance in Sport and the Dynamics of Talent Development. Sports Medicine, 40(4), 271-283. doi:10.2165/ 11319430-000000000-00000

Prieto, J.M. (2017). Experiencia deportiva, ansiedad y motivación en corredores populares. Cuadernos De Psicología Del Deporte, 17(1), 51-58. Recuperado de https://revistas.um.es/cpd/ article/view/

Robbani, F. I., y Dimyati. (2019). Psychological Skills of Soccer Players Based on Gender Perspective. Journal of Physical Education, Sport, Health and Recreation. 8(1), 44-50.

Serra-Olivares, J., Prieto-Ayuso, A., Pastor-Vicedo, J. C., \& González-Víllora, S. (2019). Propuesta de evaluación multidisciplinar del talento de jóvenes futbolistas (Proposal for a multidisciplinary assessment of talented football players). Retos, 38(38), 782-789. Recuperado de https:// recyt.fecyt.es/index.php/retos/article/view/73118

Smith, R., Schutz, R., Smoll, F., y Ptacek, J. (1995). Development and Validation of a Multidimensional Measure of SportSpecific Psychological Skills: The Athletic Coping Skills Inventory-28. Journal of Sport \& Exercise Psychology, 17, 379-398. doi:10.1123/jsep.17.4.379

Stambulova, N. (2003). Symptoms of a crisis-transition/ : A grounded theory study. In Årsbok/ : Svensk idrottspsykologisk förening, SIPF (pp. 97-109). Örebro: Svensk idrottspsykologisk förening. Recuperado de http:// urn.kb.se/resolve?urn=urn:nbn:se:hh:diva-5911

Stambulova, N., Alfermann, D., Statler, T., y Côté, J. (2009). ISSP Position stand: Career development and transitions of athletes. International Journal of Sport and Exercise Psychology, 7(4), 395-412. doi:10.1080/ 1612197X.2009.9671916

Thomas, J. y Nelson, J. (2007) Introdución a la investigación en actividad física. Métodos de investigación en actividad física, Paidotribo, Barcelona.

Torreblanca-Martínez, V., Arráez, A., Otero-Saborido, F. M., \& González-Jurado, J.A. (2017). Características antropométricas $\mathrm{y}$ de rendimiento físico en futbolistas sub-11 según el nivel competitivo (Anthropometric and fitness performance characteristics in under-11 football players by competitive level). Retos, (34), 146-149. Recuperado de https://cutt.ly/ ddEyYhP

Torregrossa, M., Chamorro, J., y Ramis, Y. (2016). Transición de júnior a sénior y promoción de carreras duales en el deporte: una revisión interpretativa. Revista de Psicología Aplicada Al Deporte y Al Ejercicio Físico, 1. doi:10.5093/rpadef2016a6 ÿbWeinberg, R. S. y Gould, D. (2014). Foundations of Sport and Exercise Psychology, 6E. Human Kinetics.

Wylleman, P., Reints, A., \& De Knop, P. (2013). Adevelopmental and holistic perspective on athletic career development. In P. Sotiaradou, \& V. D. Bosscher (Eds.), Managing High Performance Sport (pp. 159-182). (Foudations of Sport Management). New York: Routledge. 